\title{
Rapid Response Quality Control Service for the Laser Ranging Tracking Network
}

\author{
Toshimichi Otsubo • Horst Müller • Erricos C. Pavlis • \\ Mark H. Torrence • Daniela Thaller • Vladimir D. Glotov • \\ Xiaoya Wang • Krzysztof Sośnica • Ulrich Meyer • Matthew J. Wilkinson
}

Received: date / Accepted: date

T. Otsubo

Geoscience Laboratory, Hitotsubashi University

2-1 Naka, Kunitachi, Tokyo, 186-8601, Japan

E-mail: t.otsubo@r.hit-u.ac.jp

\section{H. Müller}

Deutsches Geodätisches Forschungsinsitut

Technische Universität München

Arcisstraße 21, 80333 München, Germany

E-mail: horst.mueller@tum.de

E. C. Pavlis

Joint Center for Earth Systems Technology

1000 Hilltop Circle, Baltimore, MD, 21250, USA

E-mail: epavlis@umbc.edu

M. H. Torrence

Stinger Ghaffarian Technologies Inc.

7701 Greenbelt Road, Greenbelt, MD, USA

E-mail: mark.h.torrence@nasa.gov

D. Thaller

Bundesamt für Kartographie und Geodäsie

Richard-Strauss-Allee 11, 60598 Frankfurt am Main, Germany

E-mail: daniela.thaller@bkg.bund.de

V. D. Glotov

Central Research Institute of Machine Building

4 Pionerskaya str., 141070 Korolyov, Moscow region, Russia

E-mail: vladimir.glotov@mcc.rsa.ru

X. Wang

Shanghai Astromomical Observatory

80 Nandan Road, XuHui District, Shanghai 200030, China

E-mail: wxy@shao.ac.cn

K. Sośnica

Institute of Geodesy and Geoinformatics, Wroclaw University of Environmental and Life Sciences

Grunwaldzka 53, 50-357 Wroclaw, Poland E-mail: krzysztof.sosnica@igig.up.wroc.pl

U. Meyer

Astronomical Institute, University of Bern

Sidlerstrasse 5, 3012 Bern, Switzerland

E-mail: ulrich.meyer@aiub.unibe.ch

\begin{abstract}
Quality control systems for satellite laser ranging observations have been developed at a number of analysis institutes worldwide, using various software packages of precise orbit determination and data analysis. Satellite laser range observations, primarily from the two LAGEOS satellites but also from other satellites in low-Earth-orbits and up to GNSS altitude, are being processed on a sub-daily to weekly basis. The generated quality control reports are widely used to detect various kinds of problems and quickly provide anomalous information to laser ranging stations. They have been effective in shortening the time to return to normal when anomalous data are detected and in quantifying the performance of laser ranging stations. Consequently, a rapid feedback loop has now been incorporated in the modern satellite laser ranging operation.
\end{abstract}

Keywords Satellite laser ranging - Precise orbit determination · Quality control · Data anomaly detection

\section{Introduction}

The majority of currently active Satellite Laser Ranging (SLR) stations have achieved sub-centimeter precision in the two-way range measurements between a ground station and satellites at various altitudes. SLR has continuously contributed to global geodetic products, orbit validation and other areas, and, as a result, the highquality range measurements in the optical regime have attracted many users.

M. J. Wilkinson

NERC Space Geodesy Facility

Herstmonceux Castle, Hailsham, East Sussex, BN27 1RN, UK

E-mail: matwi@nerc.ac.uk 
The quality control process, the topic of this paper, plays a key role in the operation of the SLR network. No systems are faultless especially for SLR because every SLR system is essentially different from others.

When an SLR station tracks a satellite and uploads the data, it cannot fully guarantee their quality. Stations have to calculate the root mean squares (RMS) of residuals generated during the normal-point formation process ${ }^{1}$ (compressing all shot-by-shot data points to be represented by a single point per few seconds to a few minutes) so that they can reject obvious outliers. However, it is simply a statistical dispersion in its own observations, and generally speaking, it is not possible to locally assess the accuracy of their own observation data even at a meter or 10-meter level. Hence SLR stations need some feedback about the data quality based on independent analysis. This paper discusses the activities in the frame of the International Laser Ranging Service (ILRS) (Pearlman et al., 2002) to generate and disseminate quality control information to the tracking stations.

In the early stage of SLR, the observation data were recorded on magnetic tapes and it took usually a few weeks or even months to get them analyzed. When a problem occurred at a station, therefore, the anomalous data continued flowing in for a long time. By early $1990 \mathrm{~s}$, however, it became possible for the analysts to obtain the SLR data and provide feedback to the stations within a few days (Eanes et al. 1994; Ourensma and Noomen, 1998). This benefits from the progress of computer networking. Currently, ILRS tracking stations are encouraged to release the SLR data just after the end of the pass, usually within a few hours. We can thus apply a quick-look routine analysis within a day or even less.

There are a number of ongoing activities on SLR quality control in SLR analysis institutes, such as Glotov et al. (2004), Pavlis and Kuzmicz-Cieslak (2007), Otsubo et al. (2008), Müller et al. (2013). In this paper, we shall focus on common procedures and worldwide collaboration, without going into the analysis details of each institute.

\section{Data Flow}

Continuous information flow in the SLR quality control procedure is outlined in Fig. 1. After the tracking data are transferred from each station to one of the two ILRS Operations Centers, data files are promptly organized and archived at the ILRS Data Centers after applying

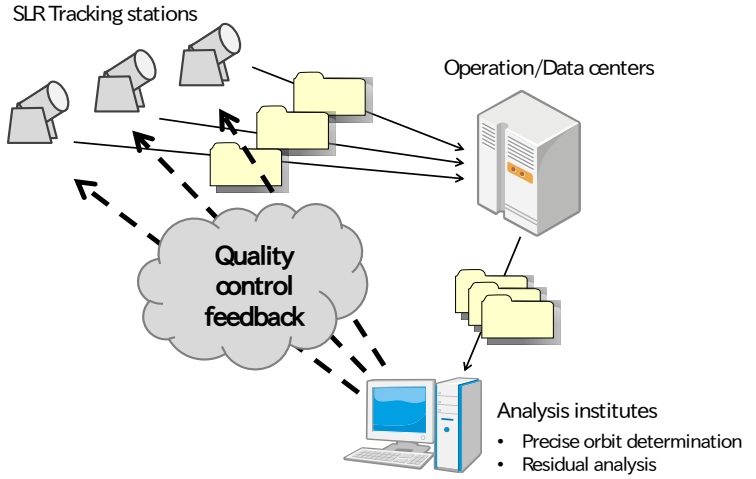

Fig. 1 SLR data flow in quality control sequence.

a format check and some nominal verification of the reported values in various fields (Noll et al. 2018).

The analysis institutes listed in Table 1 have routinely contributed to the quality control of the SLR data as of 2018. They routinely fetch the SLR data files from the Data Centers and pass them through a data reduction and orbit determination process to see how the observations fit the reference orbits. As shown in Fig. 1, the quality control institutes send quality control feedback to the tracking stations. In particular, when anomalous observations from a certain station are detected, the station should be notified.

Accuracy and reliability are the most important. Each institute has their own analysis software as listed in Table 1 and strive to improve its physical models and algorithms. Some of them have also benefited from long-term intercomparison tests through the Analysis Standing Committee of the ILRS (Luceri, 2018a). The analysis reports should be reliable and the analysis institutes make every efforts to avoid activating a false alarm.

Rapidness is also an important factor. Table 1 tells that the quality control analysis sequence is executed every day or every week. There are a couple of institutes that update the analysis reports even more frequently.

The results and products from these institutes are described in the next section.

\section{Quality Control}

\subsection{Pass-by-pass analysis}

Observational errors can be classified into two groups: random and systematic errors. The rapid quality control services for SLR are mainly focused on the detec-

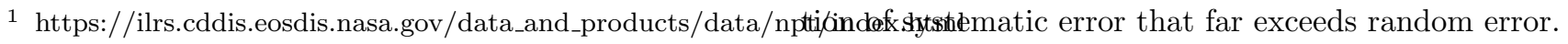


Table 1 Analysis institutes providing quality control information.

\begin{tabular}{|c|c|c|c|c|c|}
\hline Institute & Software & Output & Satellites & Update & Duration \\
\hline $\begin{array}{l}\text { Astronomical Institute, Uni- } \\
\text { versity of Bern, Switzerland }\end{array}$ & Bernese 5.3 & Range bias & GNSS & Daily & 2000-present \\
\hline $\begin{array}{l}\text { Deutsches Geodätisches } \\
\text { Forschungsinstitut, Germany }\end{array}$ & DOGS 5.4 & Range \& time bias & $\begin{array}{l}\text { ETALON, LA- } \\
\text { GEOS and LEOs }\end{array}$ & 4-hourly & 2003-present \\
\hline $\begin{array}{l}\text { Hitotsubashi University, } \\
\text { Japan }\end{array}$ & $\mathrm{c} 5++\mathrm{R} 889$ & Range \& time bias & $\begin{array}{l}\text { GNSS, ETALON, } \\
\text { LAGEOS } \text { and } \\
\text { LEOs }\end{array}$ & 6-hourly & 1998-present \\
\hline $\begin{array}{l}\text { Joint Center for Earth Sys- } \\
\text { tems Technology, USA }\end{array}$ & $\begin{array}{l}\text { GEODYN II } \\
\text { and SOLVE II }\end{array}$ & $\begin{array}{ll}\text { Range } & \& \text { time } \\
\text { bias, } & \text { Residual } \\
\text { map } & \end{array}$ & $\begin{array}{l}\text { ETALON, LA- } \\
\text { GEOS and LEOs }\end{array}$ & Daily & 2007-present \\
\hline $\begin{array}{l}\text { Information-Analytical Cen- } \\
\text { ter, Russia }\end{array}$ & STARK-C 7.7 & Range \& time bias & LAGEOS & Daily & 1997-present \\
\hline $\begin{array}{l}\text { NERC Space Geodesy Facility, } \\
\text { UK }\end{array}$ & SATAN_SX & Residual plots & $\begin{array}{l}\text { ETALON and LA- } \\
\text { GEOS }\end{array}$ & Daily & 1997-present \\
\hline $\begin{array}{l}\text { Shanghai Astronomical Obser- } \\
\text { vatory, China }\end{array}$ & SHORD-II & Range \& time bias & $\begin{array}{l}\text { ETALON and LA- } \\
\text { GEOS }\end{array}$ & Weekly & 1999-present \\
\hline $\begin{array}{l}\text { Wroclaw University of Envi- } \\
\text { ronmental and Life Sciences, } \\
\text { Poland }\end{array}$ & Bernese 5.2 & $\begin{array}{l}\text { Range bias \& } \\
\text { residual plots }\end{array}$ & GNSS & Daily & 2016-present \\
\hline
\end{tabular}

Precise orbit determination is the main tool for the quality control. Every institute involved in quality control analysis has its own orbit determination software which is automatically run on a prescribed schedule. The majority of the institutes uses only SLR data to generate the reference (i.e. best-fit) orbits, but there are a couple of institutes (Astronomical Institute, University of Bern (AIUB), and Wroclaw University of Environmental and Life Sciences (WUELS)) whose reference orbits of the GNSS (Global Navigation Satellite System) satellites are generated from the microwave data.

For the purpose of quality control, the number of unknown parameters is limited. In the SLR-only analysis institutes, for instance, only orbital parameters are solved for while other parameters such as the coordinates of the stations are usually fixed to a-priori values. Therefore, the accuracy of the adopted station coordinates has a direct effect on the accuracy of quality control and it is very important, especially, in the case of new/re-installed stations, those significantly improved or those located in seismically active areas.

After iterative fits with outlier elimination, the postfit residuals of normal points typically scatter from 0.7 to $1.5 \mathrm{~cm}$ weighted RMS for LAGEOS and LAGEOS-2. The residuals scatter more, from 1 to $4 \mathrm{~cm}$ weighted RMS, for low-orbit spherical satellites such as Starlette, Stella, Ajisai and LARES. The station-dependent weights are empirically based on the quality of the station data.

The top part of Fig. 2 shows typical post-fit residuals for LAGEOS 7-day SLR data, which is taken from the latest quality control analysis at Hitotsubashi University. 11 parameters (6 orbit (position and velocity)
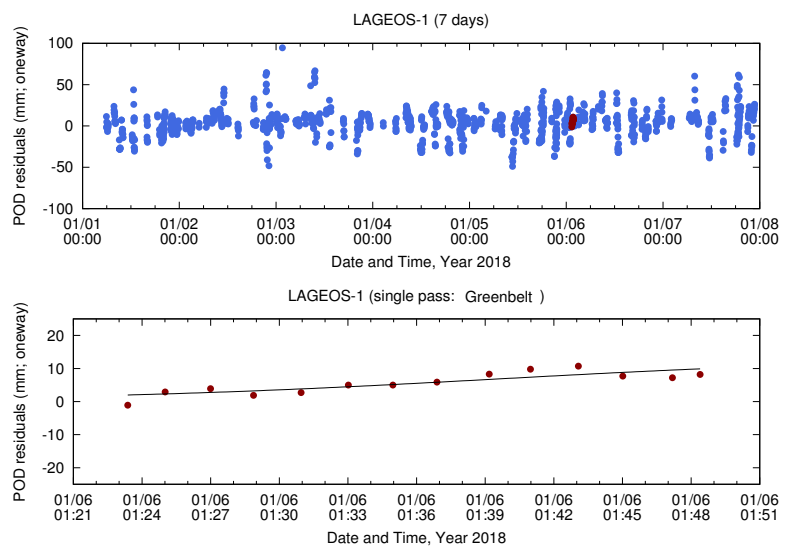

Fig. 2 A LAGEOS post-fit residual example of a good pass. Top: the whole network throughout the 7-day arc. Bottom: one of many good behaving passes.

elements and 5 empirical parameters in along-track and cross-track components) are solved for per 7-day arc. In this particular span, the weighted RMS was $1.0 \mathrm{~cm}$ after iterative removal of outliers. The remaining noise is stemmed from either imperfect physical models or observation errors. For instance, a pass observed from the Greenbelt SLR station is marked in red in both graphs of Fig. 2, and the bottom graph is simply zoomedin with a 2-parameter best-fit curve (in black) of a range bias parameter and a time bias parameter, both of which are introduced later. Most of SLR data from good performing stations align near the zero level as in this example.

If we detect observations from a station deviating far from the zero level, i.e. the reference orbit, the station is likely to have a problem in its operation. We sometimes 


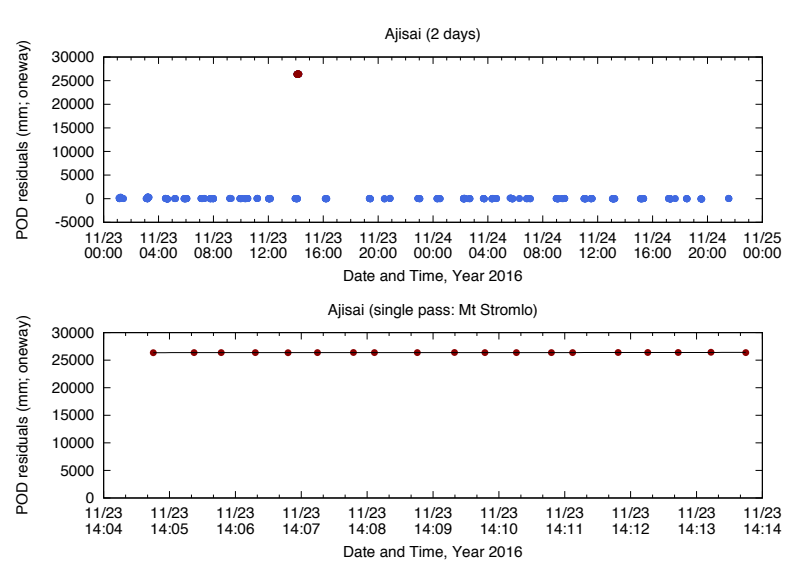

Fig. 3 An Ajisai post-fit residual example with large range bias. Top: the whole network throughout the 2-day arc. Bottom: a Mt Stromlo pass with +26 meter range bias.
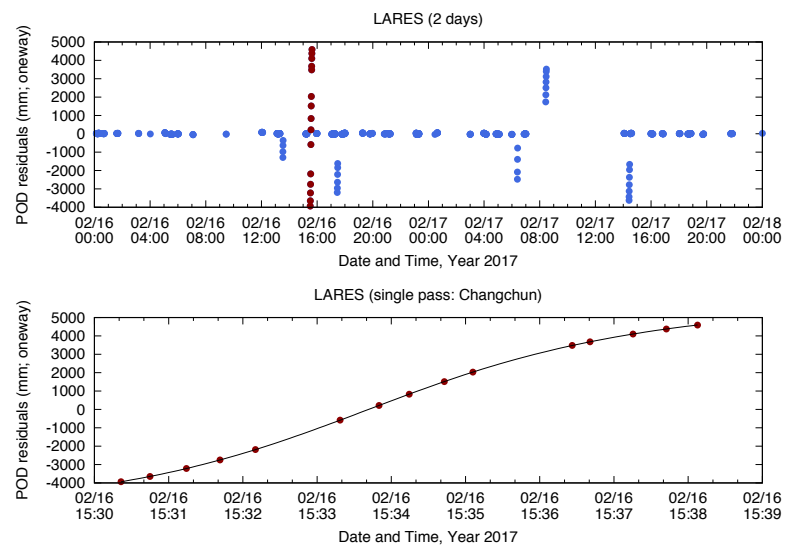

Fig. 4 A LARES post-fit residual example with large time bias. Top: the whole network throughout the 2-day arc. Bottom: a Changchun pass with +1 millisecond time bias.

see the cases like the example shown in Fig. 3 where all the SLR data in a pass are deviating greatly and consistently from the reference orbit. We also see the cases as the one displayed in Fig. 4 where the residuals show a large negative-to-positive (or opposite) trend.

These two kinds of errors are called range bias $\Delta_{R}$ and time bias $\Delta_{T}$, respectively. The majority of anomalous data falls into either of them. The conceptual diagrams for a satellite pass are shown in Fig. 5. Range bias is defined as a constant error in the range observation and expressed in a unit of length such as meter or millimeter. Time bias is defined as a constant error in the time tag and expressed in a unit of time such as microsecond or millisecond. Note that the satellites dealt within this paper orbit the Earth at the velocity of roughly 4 to $8 \mathrm{~km} / \mathrm{s}$. A one-microsecond time bias thus corresponds to 4 to $8 \mathrm{~mm}$ in the along-track component.
Given a sufficient tracking duration and a sufficient number of normal-point observations, these two bias parameters, range bias $\Delta_{R}$ and time bias $\Delta_{T}$, can be estimated from a population of post-fit residuals whose $i$-th element is defined as $y_{i}$. The observation equation is written as:

$y_{i}=\Delta_{R}-\dot{\rho}_{i} \Delta_{T}+\epsilon_{i}$

where $\dot{\rho}_{i}$ is the range rate for the $i$-th observation, and $\epsilon_{i}$ is the error in the $i$-th observation. This equation should be stacked for the number of observations per pass, and the two bias parameters $\Delta_{R}$ and $\Delta_{T}$ can be estimated in a standard least square adjustment.

In reality, it is sometimes difficult to estimate both of the two bias parameters when the tracking duration is too short or the number of observations is too few. In such cases, most analysis institutes provide only range bias, i. e. the simple mean of the residuals.

Fig. 6 is an example of quality control reports of Hitotsubashi University. The institutes in Table 1 whose output is 'Range \& time bias' also generate and update numerical tables in similar formats. Each line corresponds to one pass. The column of 'rb' corresponds to estimated range bias, and that of ' $t b$ ' to estimated time bias, both of which are followed by their formal errors of Eq. 1 in parentheses. In this case of the Yarragadee station, the error in the orbit models, about $1 \mathrm{~cm}$ RMS, well exceeds the precision in the normal-point observations, about a few mm RMS, and therefore the bias values given here scatter more than the actual observation precision. This is the typical case for good observations from good stations. If these bias values showed a huge jump which is significantly larger than its estimated error, and if the bias persisted for multiple passes, it is likely that the station has a problem with their equipment, software or operation.

In addition to numerical tables, some institutes provide graphical information. Various kinds of plots are being generated in Deutsches Geodätisches Forschungsinsitut, Technische Universität München (DGFI-TUM). One example in Fig. 7 shows LAGEOS range bias estimates of the Herstmonceux station. Users can visually see long-term behavior of their daytime observations and the nighttime observations. In this particular

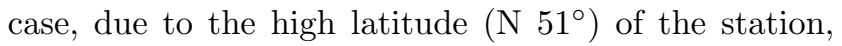
the amount of day/night passes has a clear annual pattern, and the day-night differences of the moving average curve nicely stay within $3 \mathrm{~mm}$. Graphical plots are clearly more comprehensible and more informative for users.

Historically, only the two LAGEOS satellites, i.e., LAGEOS and LAGEOS-2, had been applied to quality control since they are primarily used for geodetic anal- 


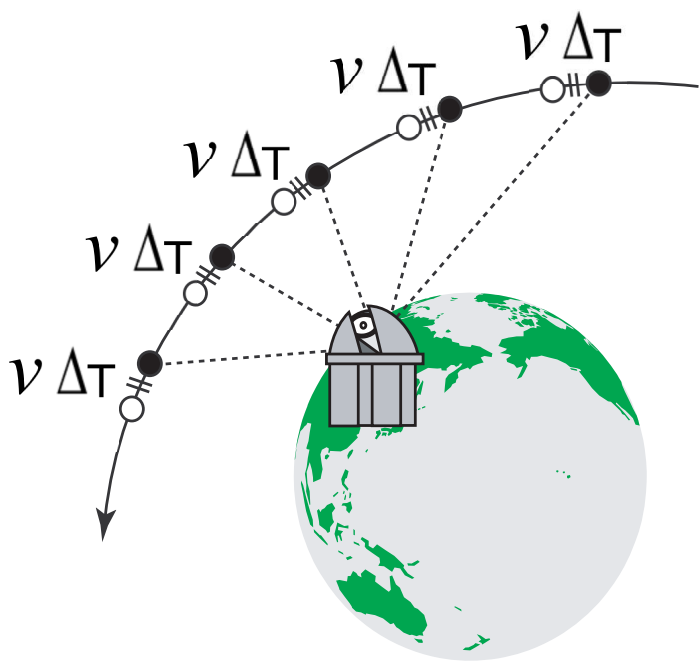

true orbit

\section{observation}

Fig. 5 Range bias (left) and time bias (right). Illustrated are positive biases in both cases. $v$ is the velocity of the satellite.
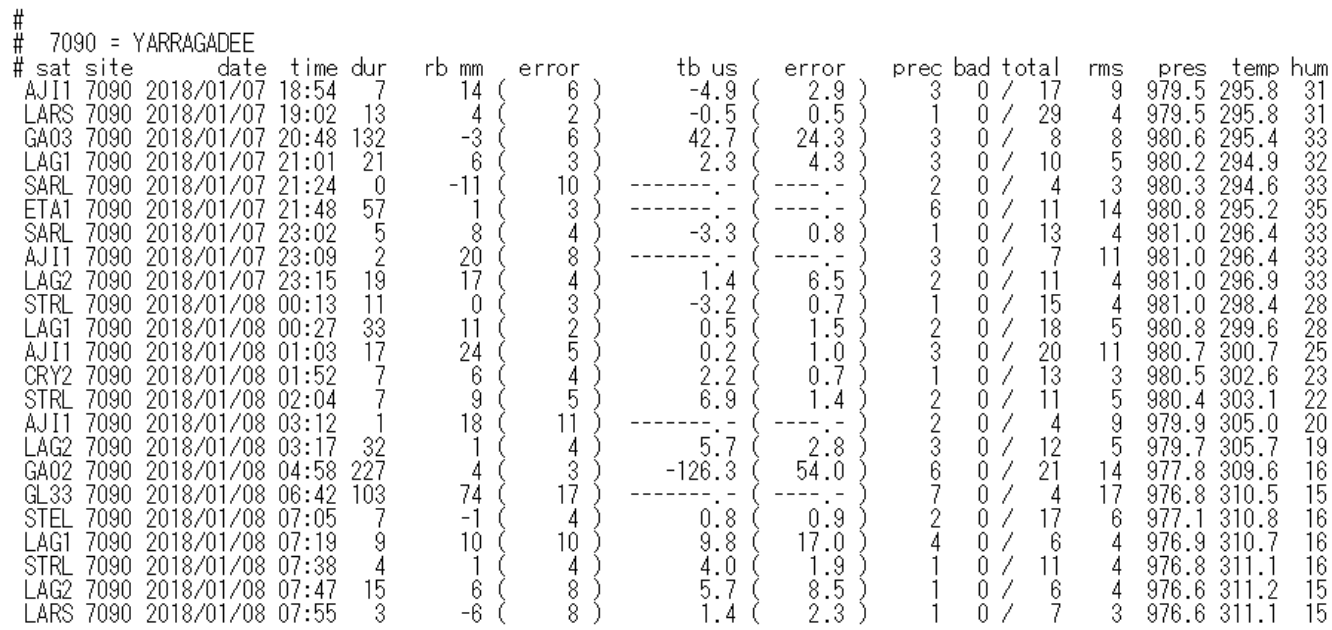

$\begin{array}{rr}\text { sdel ay shft } \\ 13079 & 0 \\ 13079 & 0 \\ 21896 & -0 \\ 13082 & 0 \\ 13082 & 0 \\ 21896 & -0 \\ 13083 & 1 \\ 13083 & 1 \\ 13083 & 1 \\ 13084 & 0 \\ 13084 & 0 \\ 13084 & 0 \\ 13084 & 0 \\ 13084 & 0 \\ 13084 & 1 \\ 13084 & 1 \\ 21897 & 1 \\ 21898 & 0 \\ 13086 & 2 \\ 13086 & 2 \\ 13086 & 2 \\ 13086 & 2 \\ 13086 & 2\end{array}$

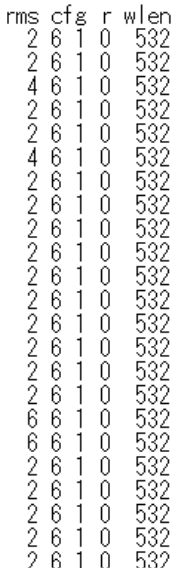

Fig. 6 Example of a quality control report (excerpted from the 16 January 2018 report issued by Hitotsubashi University). Listed are: satellite name, station ID, observation date and time, pass duration (in minutes), range bias and its estimation error (in $\mathrm{mm}$ ), time bias and its estimation error (in microseconds), estimated precision of normal point (in mm), rejected and total data points, precision of raw ranging (in $\mathrm{mm}$ ), pressure (in $\mathrm{hPa}$ ), temperature (in Kelvin), humidity (in percents), applied system delay (in $\mathrm{mm}$ ), delay shift (in $\mathrm{mm}$ ), precision of calibration ranging (in $\mathrm{mm}$ ), two ILRS configuration indicators, release flag and laser wavelength (in micrometers), from left to right.

yses, their characteristics are well understood and their orbit are less prone to poorly known physical process (e.g. atmosphere and earth gravity field). Analysis institutes have gradually added high-orbit and low-orbit satellites to the analysis of quality control reports. Despite the fact that the orbit determination precision of these satellites is not as good as that of the LAGEOS satellites, the use of a wide variety of satellites has great advantages as we explain in the following.
Firstly, the timing of problem occurrence can be exactly and reliably identified because of the increased number of passes. Secondly, we can detect problems that are not possible to be detected by analyzing only LAGEOS data because some stations have multiple hardware configurations for different satellite altitudes and because some stations are more productive in lower or higher satellites than LAGEOS. Thirdly, a range-dependent bias, often proportional to the range resulting from a 


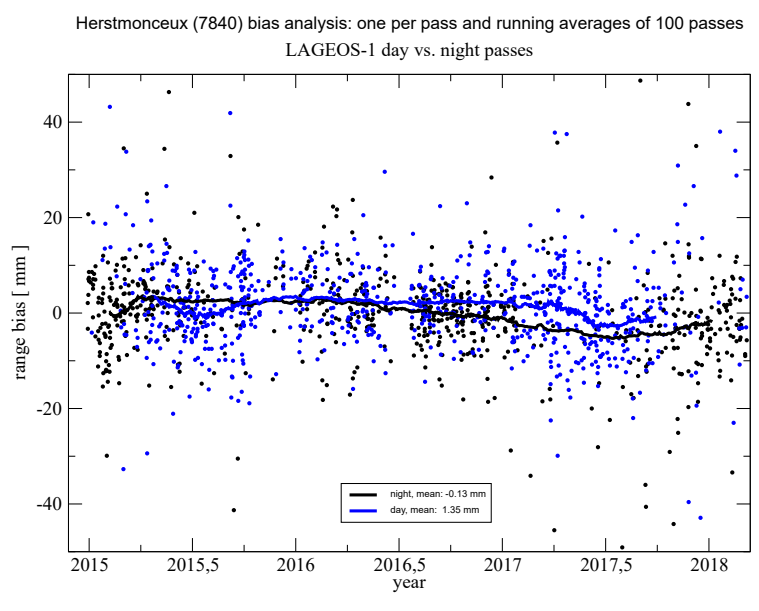

Fig. 7 A plot example of LAGEOS range bias of the Herstmonceux station, UK. DGFI-TUM routinely generates not only numerical tables but also these plots for all stations.

problem in the clock frequency, can be clearly identified.

Sufficient coverage during a pass is critical to this kind of quality control. With just a few normal-point observations over a short time, it is not possible to estimate reliably the two parameters. As a consequence, we often estimate the range bias only. It should be noted that long duration SLR passes are important for data quality assessment. In particular, a horizon-to-horizon pass duration of a high-orbit target, such as the two ETALON satellites and the GNSS satellites, is typically 5 to 8 hours. We need SLR coverage of at least 1 hour to separate the two bias parameters. However, when the SLR coverage during a pass is short, we often find that the time bias parameter cannot be determined at all, or are not well determined, for high-orbit satellite passes.

It is no surprise therefore that AIUB provides only the range bias estimates in their routine reports dedicated to the high-orbit GNSS satellites. Reference orbits in the AIUB quality control analysis are based only on GNSS microwave data. In 2017, the number of registered passes to GNSS was almost four times larger than the number of LAGEOS passes, which allows for a rigorous analysis of SLR biases at high-altitude targets. The AIUB orbits come from the analysis at the International GNSS Service processing at the Center for Orbit Determination in Europe (Dach et al. 2009). Consequently, the reported SLR bias values include the systematic offset between the two independent techniques: Thaller et al. (2011) studied the impact of mismodelled antenna phase center issues and unconsidered range bias, and Sośnica et al. (2015) investigated the pattens of SLR residuals in relation to SLR system types and onboard retroreflector array types.

As a result of there being a lot more SLR satellites in space, a ground station can now see 10 SLR satellites or even more above the horizon while it can track only one satellite at a time. The stations are encouraged to switch targets frequently (often called "interleaving mode") rather than solid tracking from the beginning to the end of the pass, and, by doing so, they would obtain SLR data from more satellites in an efficient way.

\subsection{Residual plots}

Quality control information is reduced to just one line per pass in the previous subsection. We have shown several normal-point residual graphs (Figs. 2, 3 and 3), but they are not routine products. Residual graphs are indeed more informative and useful for a precise understanding of the behavior of observation data. NERC Space Geodesy Facility (SGF) and WUELS provide online residual graphs so that the users can visually see the precise details of normal-point residuals such as outliers and trends.

The SGF updates on its website each day interactive normal-point residual plots of LAGEOS and Etalon normal points submitted globally over the past 7 days as a by-product of its routine analysis center activity. The SGF also separately carries out daily "short-arc" quality control, which extracts and plots a commonview pass from multiple stations located nearby (Sinclair and Appleby, 1993). Through these plots available online, we see how each normal point behaves with respect to the reference orbit. An example is shown in Fig. 8, which shows four stations tracking LAGEOS. In this particular case, the LAGEOS satellite flies firstly over Europe and then over the USA. This plot would suggest a range bias is present in the Borowiec range measurements. These plots show solid/sparse tracking patterns and the behavior of each normal point. For instance, in the past, we frequently saw outliers at the beginning or at the end of a pass, but it is rare today thanks to alerts from this kind of service.

WUELS provides online tools for an interactive generation of SLR residual plots of GNSS satellites, based on the microwave orbits processed at AIUB in the framework of the IGS multi-GNSS Experiment (Prange et al. 2017). More than 50 active GNSS satellites are today tracked by the SLR stations, which results in a substantial number of SLR observations. The WUELS service allows for the residual analysis with respect to the date and time of data acquisition, differences from nighttime and day-time tracking, dependencies of residuals as a function of the elevation and azimuth angles. All 


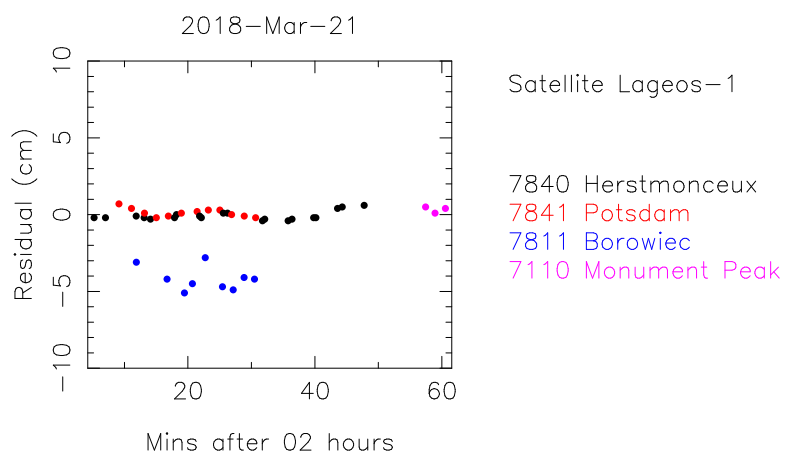

Fig. 8 A residual plot example of a LAGEOS-1 commonview pass from Europe-USA region. This is taken from NERC SGF's daily short-arc quality control.

of them help in identification of various systematics. The analyses can be performed for individual stations and satellites or for a user-defined group of satellites or stations.

\section{Integration and communications}

4.1 Quality control feedback

All of the quality control activities described in the previous section are intended to give prompt feedback to the tracking stations. Some stations are routinely watching the quality control reports, and the analysis institutes also send an alert message when they detect anomalous observations.

All of the analysis institutes in Table 1 routinely update the quality control reports on their websites and/or make them available through the ILRS archive and the ILRS mailing list. The long-time records of daily and weekly reports are archived not just at each institute but also aggregated on the ILRS Data Center, at Crustal Dynamics Data Information System (CDDIS) of National Aeronautics and Space Administration (NASA). The URLs of the SLR quality control services are collectively listed in Table 2 .

Users can see the quality control results from multiple institutes at once. Range bias estimates of the two LAGEOS satellites from multiple institutes are easily compared by the combined range bias reports weekly sent by AIUB. Each line of the combined report contains range bias estimates from five analysis institutes. Laser station operators can verify the reliability by checking whether an anomalous result is detected by all institutes or just by one or few institutes. It also helps analysts to see whether their results are in harmony with others.

It has been common to look at the text-based reports in the analysis institutes and also in the tracking

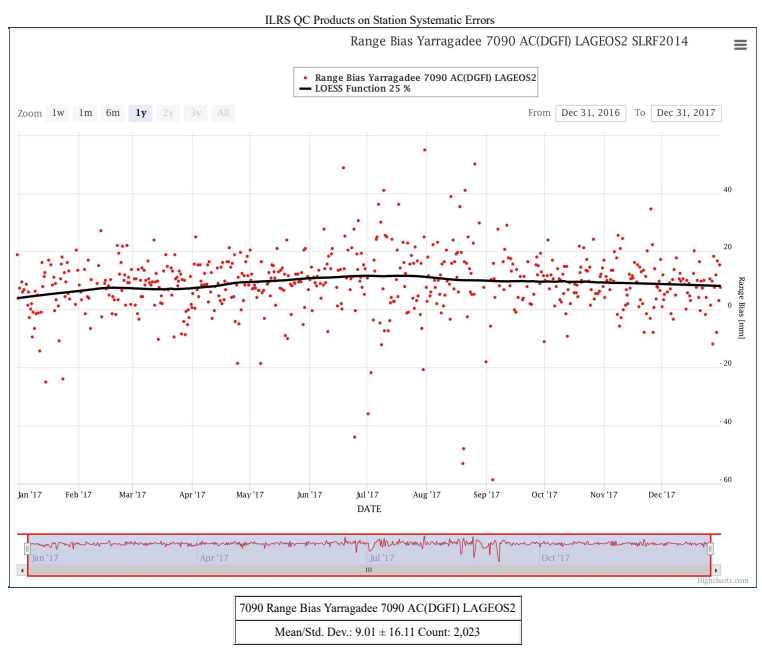

Fig. 9 A range bias plot example of LAGEOS-2 range bias estimates of Yarragadee in 2017, plotted by JCET's online interactive tool. DGFI-TUM's quality control results are chosen in this case.

stations, but an interactive graphical web tool helps more. The time series of pass-by-pass range bias, time bias and other quality control parameters can flexibly be plotted in a web browser using the QC-viewer, a service provided by the Joint Center for Earth Systems Technology (JCET) as shown in Fig. 9. This service helps users to visually understand the long-term and short-term behaviors of the quality of a certain station, optionally with a smoothing curve. The user has various options to choose from, such as the satellite, the station, the quantity of interest, the axis scales and so on, for customized graphs. In addition to JCET's own analysis results users can also choose those from the other QC institutes appearing on Table 1.

The analysis institutes look through the quality control reports. When a series of anomalous observations are clearly detected, it is very likely stem from the observation itself, not from the analysis side. A warning message is then sent to the station. The whole quality control procedure in the analysis and the data transfer is almost automated, but manual handling is still to some extent involved in detecting anomalous data and also notifying the stations.

We usually use email for communication with stations. In particular, DGFI-TUM, Hitotsubashi University and JCET have been active so far in sending alerts to the worldwide stations. In June 2011, the analysis institutes agreed to use the newly established "RapidServiceMail" mailing list implemented at DGFI-TUM, which is useful to notify and archive the anomaly detection events among analysis institutes.

In addition to the worldwide services, there are also regional activities for SLR quality control. For instance, 
Table 2 URLs for the quality control services.

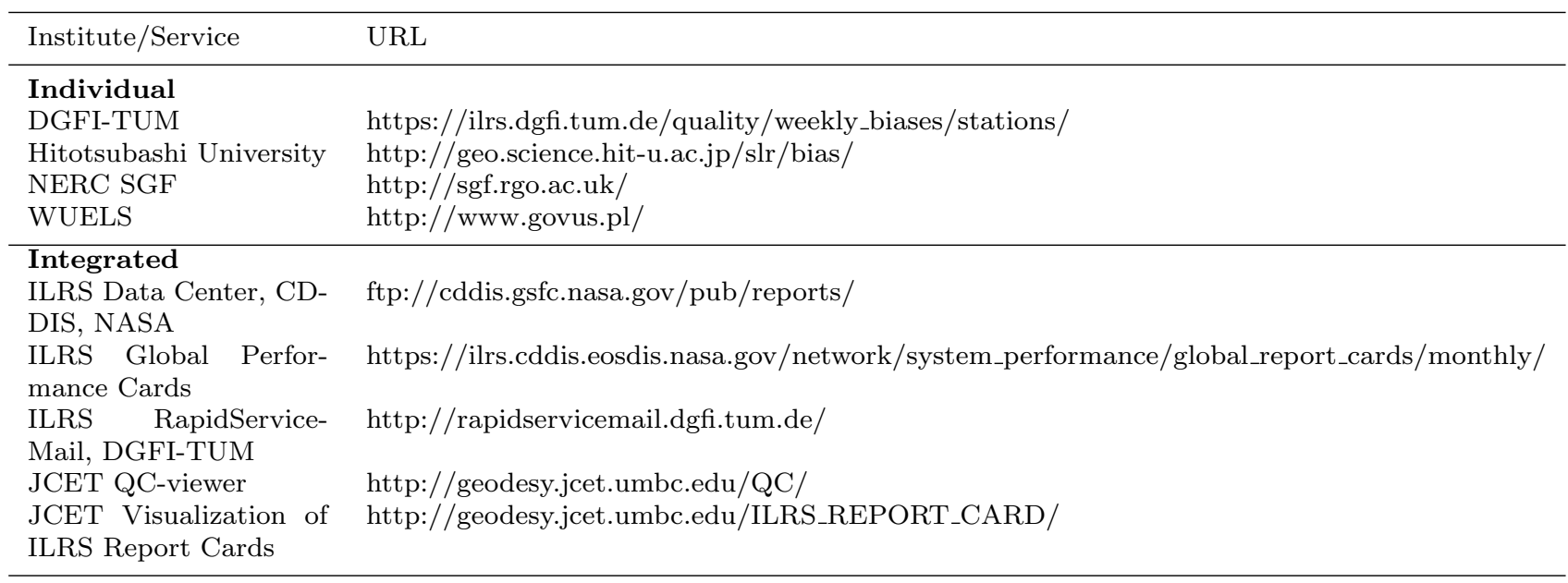

the Information-Analytical Center of Russia provides weekly feedback to stations of the Russian SLR network in the Russian language. Their weekly reports sent to the Russian SLR stations contain quantitative and qualitative statistical information customized for the Russian network, which includes the widely-available pass-by-pass quality control (range bias and time bias) reports of the LAGEOS satellites.

It is now possible to detect and notify a problem within a day. If a problem can be subsequently corrected afterwards, the station is strongly advised to resubmit the corrected observations with an incremented release flag. We have seen that most of the problems can be solved instantly or within a few days. It minimizes the time that large bias is present in the data.

Pass-by-pass analysis reports from the analysis institutes are gathered at the ILRS Central Bureau where a long-term statistics report is published from both qualitative and quantitative aspects. The "SLR Global Performance Report Card" covers the latest 12 months and is currently updated every month (every 3 months until 2012). It consists of two tables: one is for quantity and the other is for quality. The first table provides the ranking by the number of passes, and the number of normal point observations, for different kinds of satellites. The second table provides a summary of the five quality control reports providing a pass-by-pass bias analysis of the LAGEOS satellites. The results are averaged over time to produce a stability measure in two ways: a pass-by-pass range bias variation over the last three months ('short-term' stability) and a monthlyaverage range bias variation using over the last twelve months ('long-term' stability). These tables are helpful to compare a station's performance with respect to others and often encourage the improvement of the quality and quantity of their data. They are also useful to mon-

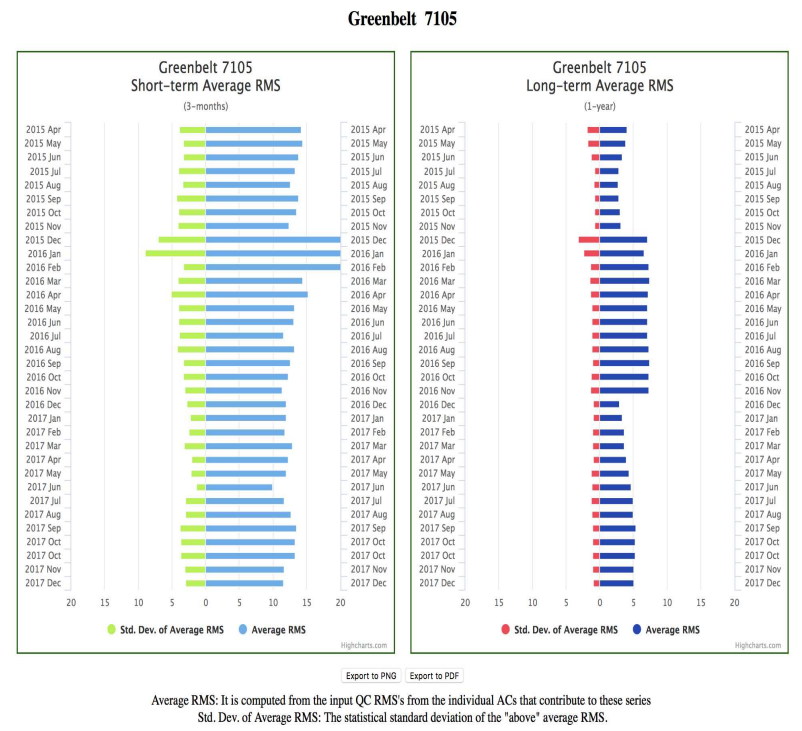

Fig. 10 Rendering of the short-term and long-term stability measure from the quarterly report card of Greenbelt using the online interactive tool developed by JCET.

itor the evolution of a station's performance over time. To facilitate these comparisons, JCET has established a service that archives all report cards' data online and allows the user to create plots of these statistics over time for each station of interest (Fig. 10). The results plotted are the average of the available short-term or long-term stability estimates from all quality control centers and the standard deviation of that estimate.

\subsection{Causes of anomalous measurements}

We often receive a reply from the station about the cause of the problem. Based on our long experience in 
interacting with the stations, we have learned that certain patterns are connected to specific causes.

The most frequent one is constant offset range bias. Some SLR laser systems transmit multiple pulses at a fixed interval within one or a few nanoseconds, where one pulse is usually predominantly stronger than others. However, especially in the case that there is more than one strong pulse, the choice of the main pulse could be wrong. The other major cause originates from calibration. It is often caused by reflection from a point different from the calibration target. If the internal system delay is wrongly measured, the SLR data are shifted by the same amount as its error. In both cases, a jump of the system delay is also observed. It is thus important to always keep an eye on the time series of the system delay measurement. Human input error during operation or data reduction can also be the cause.

Time bias is also detected almost as often as range bias. Note that the SLR observables are basically the combination of a time tag and a two-way range. Large time bias indicates a problem in time tagging. Time bias sometimes appears constant, and sometimes varying, i.e. the pass-by-pass time bias estimates gradually change over time. The problem comes from a frequency source, other timing devices and sometimes software. In the past, one second time bias was often seen after a leap second but it is rare nowadays. Extremely large time bias of one day (wrong day) and even one year (wrong year) can be also detected at times.

Meteorological sensors such as a barometer and a thermometer are sometimes found to be an error source. Atmospheric delay models are constructed as a function of meteorological data and therefore a problem in these instruments manifests itself as a range error.

Analysis institutes must pay attention not to send a false alarm. Orbit determination has not always properly converged due to an insufficient data amount, too many outliers, imperfect physical models, and orbit maneuvers. For instance, assume that a satellite is sparsely tracked even by the whole network and one productive station has large time bias, and then a well-performing station located nearby sometimes has spurious time bias estimates too. This is because time bias is inseparable from the along-track component of satellite orbits, and because large time bias can affect the orbital parameters if the actual anomalous data cannot be properly eliminated in the orbit determination stage. In another instance, the use of accurate station positions and velocities is essential for reliable and precise quality control results. Analysis institutes thus obtain, or update, the station coordinates if a station is new, has undergone significant changes, or is displaced due to earthquakes. Currently, the latest version of SLRF2014
(Luceri et al. 2018b), an extension of ITRF2014, is widely adopted for quality control analyses and frequently updated.

\section{Conclusions and ongoing/future studies}

Precise orbit determination techniques and high-speed communications have been well integrated to realize rapid quality control feedback for the SLR station network. Analysis institutes have routinely provided analysis reports in various ways and they have contributed to improving the overall quality and stability of the SLR observations.

A new ILRS study group called Quality Control Board was newly organized in December 2015. The rapid quality control activities covered in this paper are one of the core tasks in this group, and the latest news and future plans are being discussed in monthly international teleconferences.

High-quality SLR data is beneficial not only for geodesy but also for satellite altimetry, satellite navigation and any mission that relies on SLR for its precise orbits.

This paper focused on the rapid response feedback for relatively large systematic errors, but it is also possible to look into systematic observation errors/trends at the millimeter level by accumulating longer span of SLR data (Otsubo et al. 2014; Appleby et al. 2016). Both quality control methods, i.e. rapid and precise, should be utilized in a complementary fashion in the future so that systematic errors of any magnitude are eliminated from the data.

A new technology, Time Transfer by Laser Link (T2L2) demonstrated on the satellite Jason-2, makes it possible to examine the accuracy of time tagging at SLR stations (Exertier et al. 2017) with a 1 ns accuracy. This is much higher than the requirement for ordinary SLR, roughly $100 \mathrm{~ns}$ for $1 \mathrm{~mm}$ ranging accuracy, and several anomalous cases have been detected. The T2L2 mission on Jason-2 was terminated in April 2018, and the future missions are strongly desired from the viewpoint of quality control.

Acknowledgements The quality control analyses are indebted not just to the authors of this paper but to a number of researchers at the analysis institutes, such as Ms. M. Kobayashi of Hitotsubashi University, Mr. C. Schwatke of DGFI-TUM, Dr. M. Kuzmicz-Cieslak of JCET, Dr. G. M. Appleby of NERC SGF, Mr. R. Zajdel of WUELS, Dr. A. Maier and Dr. T. Schildknecht of AIUB and many others. T. Otsubo acknowledges the support of JSPS KAKENHI Grant Number 26400449. E. C. Pavlis acknowledges the support of NASA Grant NNX15AT34A. The quality control services are made up of continuous and intensive collaboration with the ILRS tracking stations and also with the ILRS Operation Centers and Data Centers. The quality control analyses in early days 
were led by Dr. R. Eanes of Center for Space Research, University of Texas, and Dr. R. Noomen of Delft University of Technology. Finally, we would like to point out that the international collaboration in this field could not be achieved at this level without Prof. W. Gurtner, AIUB, who sadly passed away in 2009 .

\section{References}

1. Appleby G, Rodríguez J, Altamimi Z (2016) Assessment of the accuracy of global geodetic satellite laser ranging observations and estimated impact on ITRF scale: estimation of systematic errors in LAGEOS observations 19932014. J Geodesy 90:1371-1388. doi:10.1007/s00190-016-0929-2

2. Dach R, Brockmann E, Schaer S, Beutler G, meindl M, Prange L, Bock H, Jäggi A, Ostini L (2009) GNSS processing at CODE: status report. J Geodesy, 83:353-365. doi:10.1007/s00190-008-0281-2

3. Eanes RJ, Bettadpur SV, Ries JC (1994) Multi-satellite Laser Range Residual Analysis for Quality Control of the SLR Network. In: Proceedings of the 9th International Workshop on Laser Ranging Instumentation, 1, pp 131-146 4. Exertier P, Bellia A, Lemoine JM (2017) Time biases in laser ranging observations: A concerning issue of Space Geodesy Adv Space Res, 60(5): 948-968. doi:10.1016/j.asr.2017.05.016

5. Glotov V, Abylchatova N, Mitrikas V, Zinkovsky M (2004) MCC analysis procedure of the SLR data quality and stations performance. In: Proceedings of the 14th International Workshop on Laser Ranging, 399-401

6. Luceri V, Pavlis EC (2018) Operational and Definitive Products of the ILRS Analysis Standing Committee. J Geodesy, in this issue

7. Luceri V, Pavlis EC, Kuzmicz-Cieslak M, Evans K, Pirri M, Bianco G (2018) The Contribution of Satellite Laser Ranging to the Development of the ITRF2014. J Geodesy, in this issue

8. Müller H, Bloßfeld M (2013) Quality and possible improvements of the official ILRS products. In: Proceedings of the 18th International Workshop on Laser Ranging

9. Noll CE, Horvath J, Müller H, Ricklefs R, Schwatke C, Torrence M, Information Resources Supporting Scientific Research for the International Laser Ranging Service. J Geodesy, in this issue

10. Otsubo T, Kobayashi M, Gotoh T, Kubo-oka T (2008) Daily quality control system of satellite laser ranging data for the ILRS network. Journal of Geodetic Society of Japan, 54, 2, pp. 69-79 (in Japanese)

11. Otsubo T (2014) Systematic Range Error 2013-2014. In: Proceedings of the 19th International Workshop on Laser Ranging

12. Ourensma GJ, Noomen R (1998) Quick-look analysis at Delft University of Technology: past, present and future. In: Proceedings of the 11th International Workshop on Laser Ranging, 2, 654-659

13. Pearlman MR, Degnan JJ, Bosworth JM (2002) The International Laser Ranging Service. Adv. Space Res., 30, 135143. doi:10.1016/S0273-1177(02)00277-6

14. Pavlis EC, Kuzmicz-Cieslak M (2007) Quality control of ILRS data and products, presented at ILRS Fall 2007 Workshop

15. Prange L, Orliac E, Dach R, Arnold D, Beutler G, Schaer S, Jäggi A (2017) CODE' s five-system orbit and clock solution-The challenges of multi-GNSS data analysis. J Geodesy, 91, 345360. doi:10.1007/s00190-016-0968-8
16. Sinclair AT, Appleby GM (1993) A short-arc method for determination of station coordinates and baselines applied to the Mediterranean area. In Contributions of Space Geodesy to Geodynamics: Crustal Dynamics (eds D. E. Smith and D. L. Turcotte). doi:10.1029/GD023p0389

17. Sośnica K., Thaller D, Dach R, Steigenberger P, Beutler G, Arnold D, Jäggi A (2015) Satellite laser ranging to GPS and GLONASS. J Geodesy, 89, 725-743. doi:10.1007/s00190-015-0810-8

18. Thaller, D, Dach R, Seitz M, Beutler G, Mareyen M, Richter B (2011): Combination of GNSS and SLR observations using satellite co-locations. J Geod, 85, 257-272. doi:10.1007/s00190-010-0433-z 
SLR Tracking stations

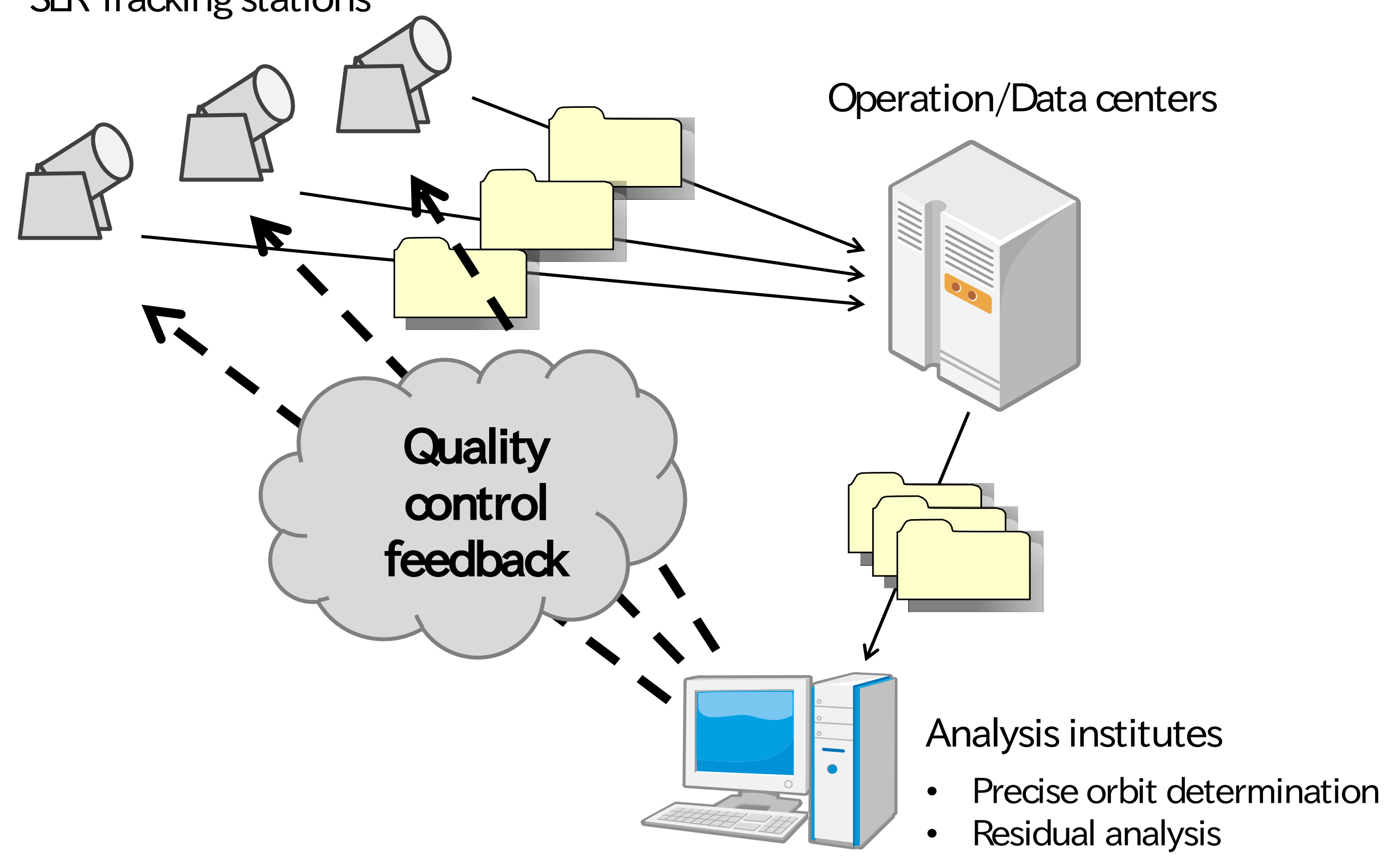


LAGEOS- 1 (7 days)

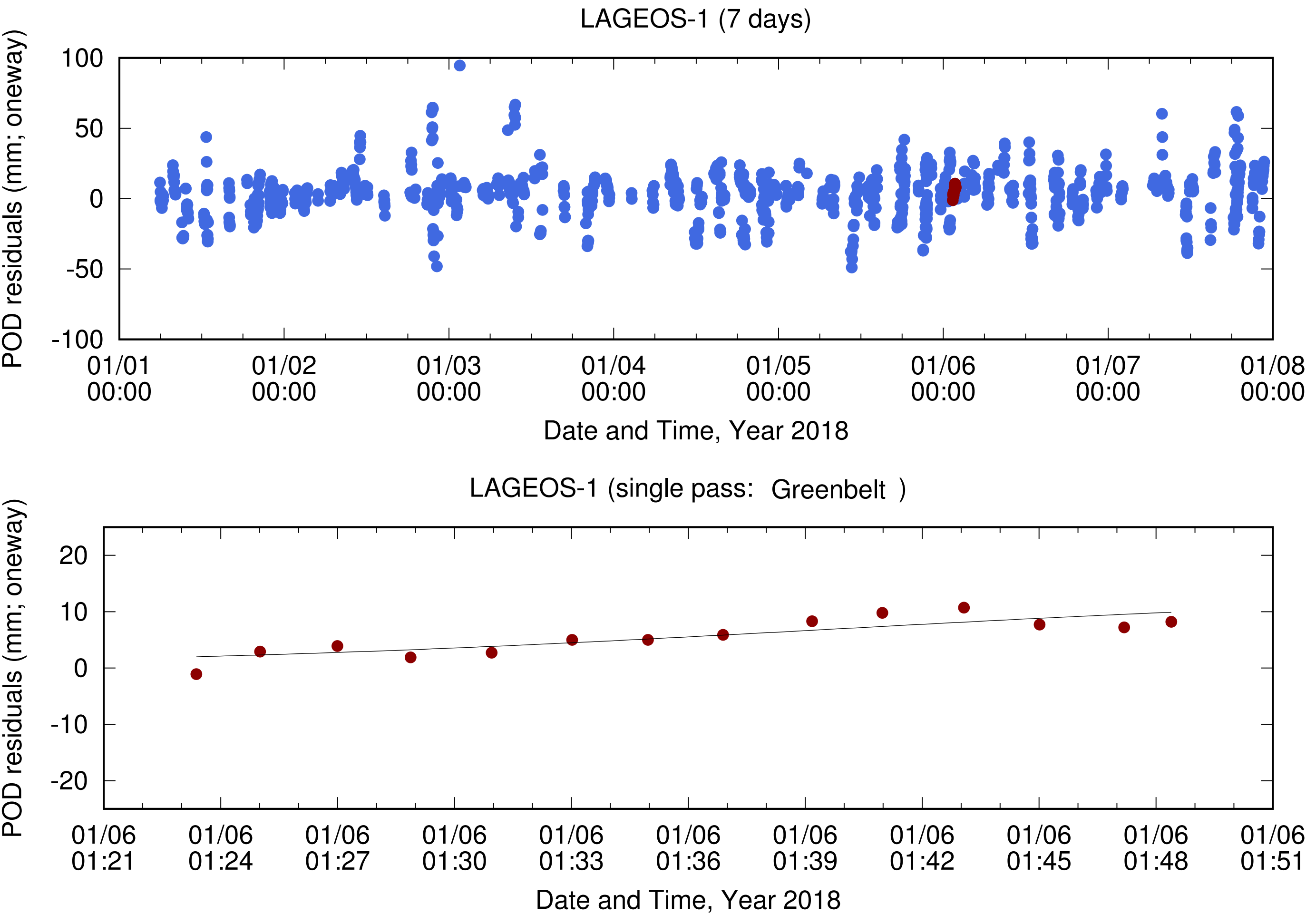


Ajisai (2 days)

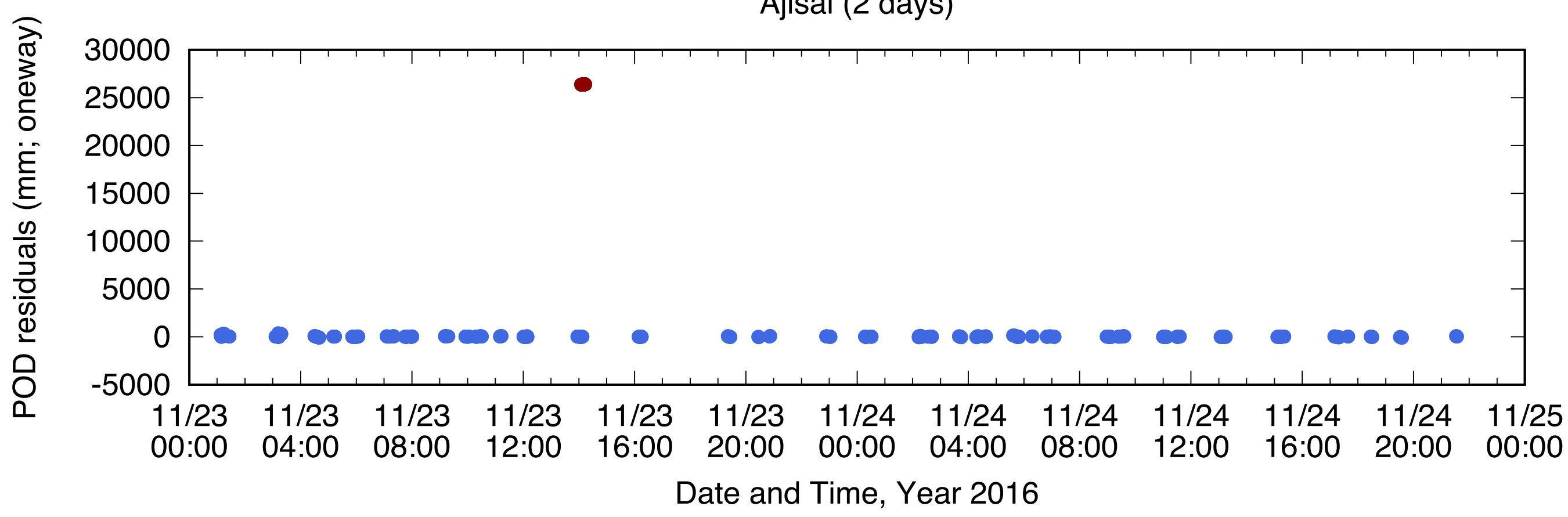

Ajisai (single pass: Mt Stromlo)

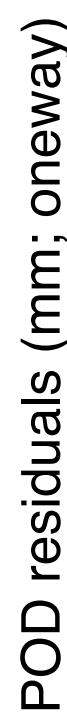

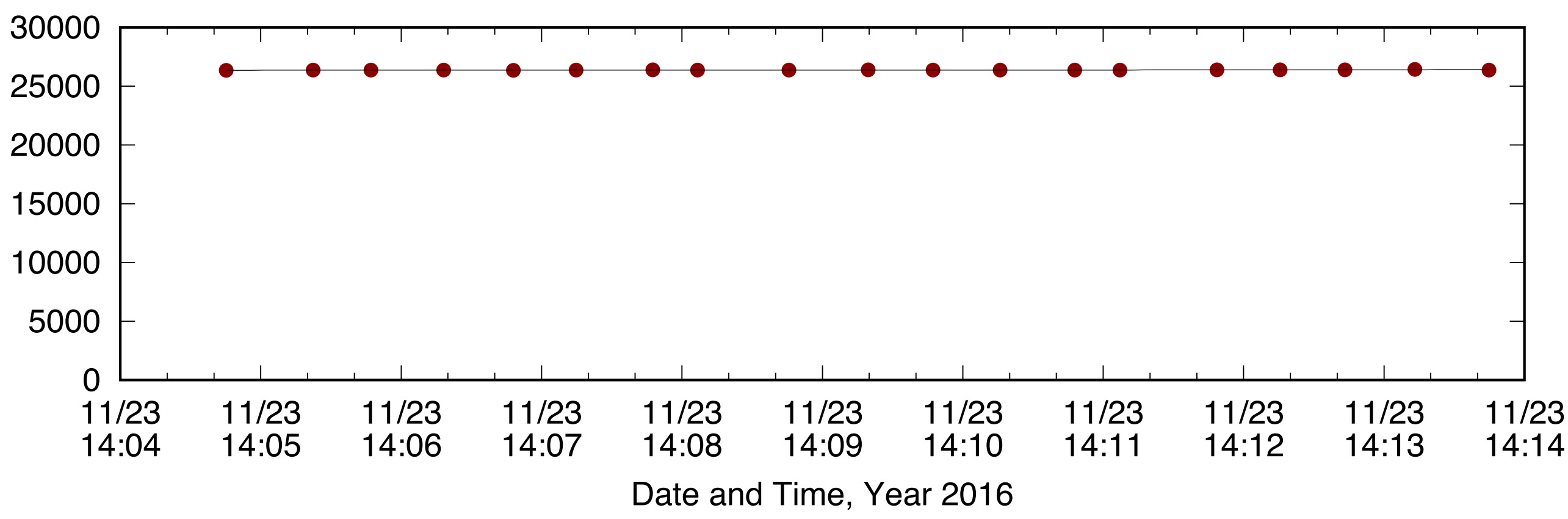




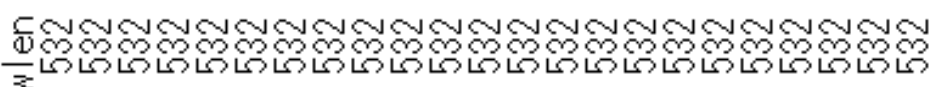

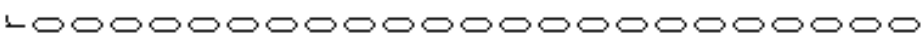

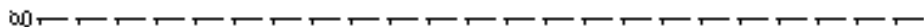

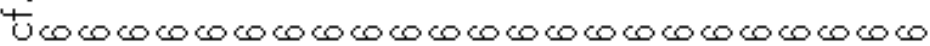
MNW 艺 $\frac{r}{n}$

उ\%

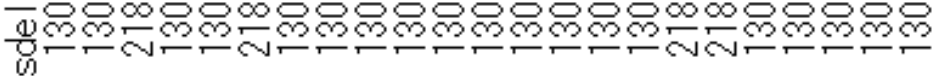

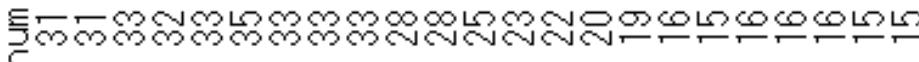

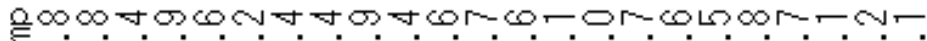

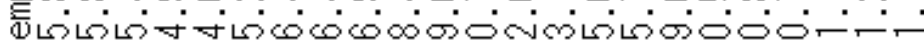

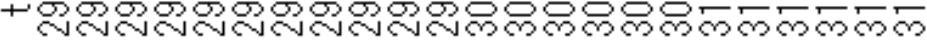

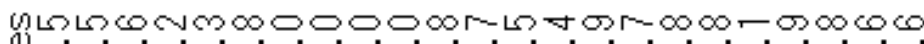

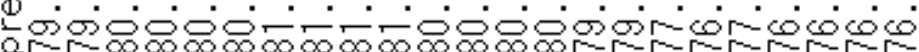
の

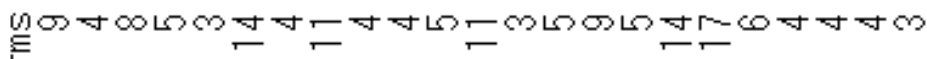
ত

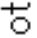

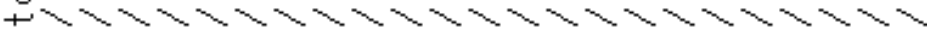

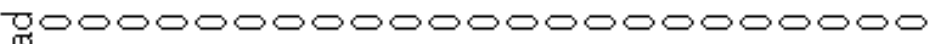


पूल- $\frac{1}{2}$

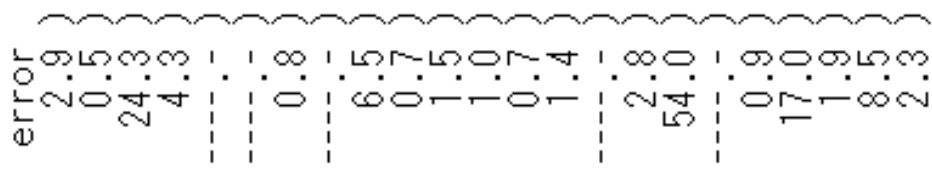

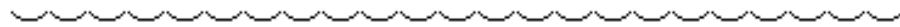

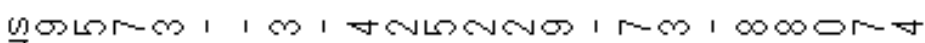

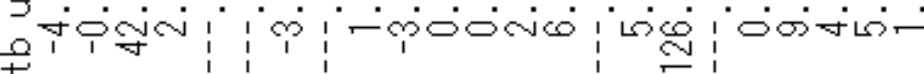
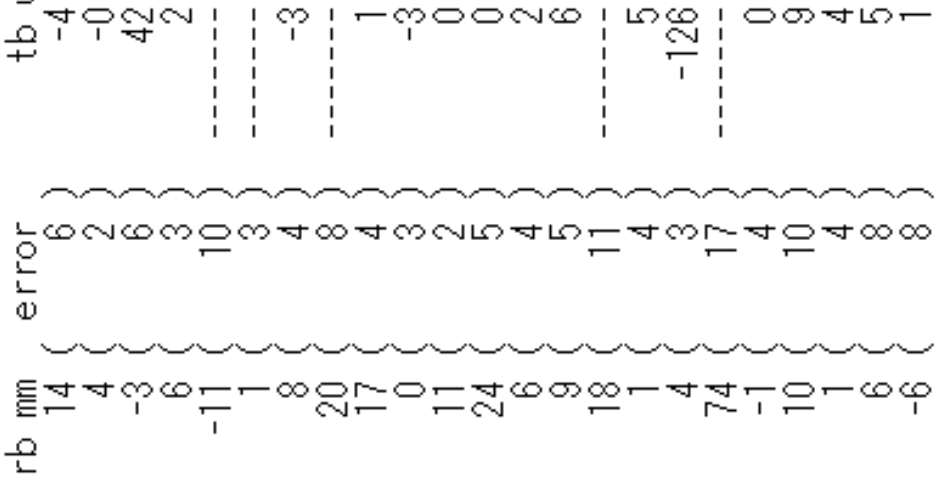
与 Uषऽ

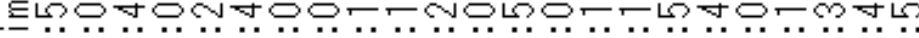

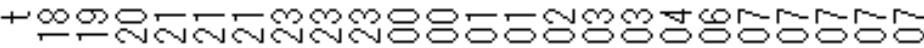
(1) 땅

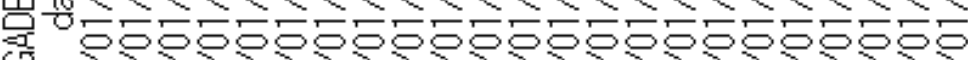
T $\infty \infty \infty \infty \infty \infty \infty \infty \infty \infty \infty \infty \infty \infty \infty \infty \infty \infty \infty)$

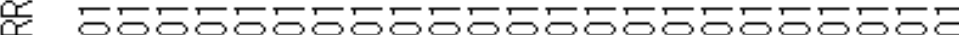

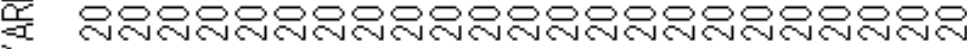
"

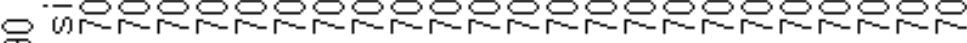
$\sigma$ 羽羽壮

एЕ

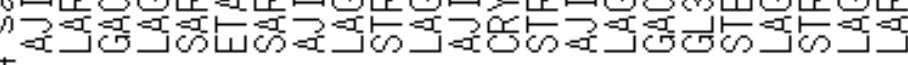


Herstmonceux (7840) bias analysis: one per pass and running averages of 100 passes

LAGEOS-1 day vs. night passes

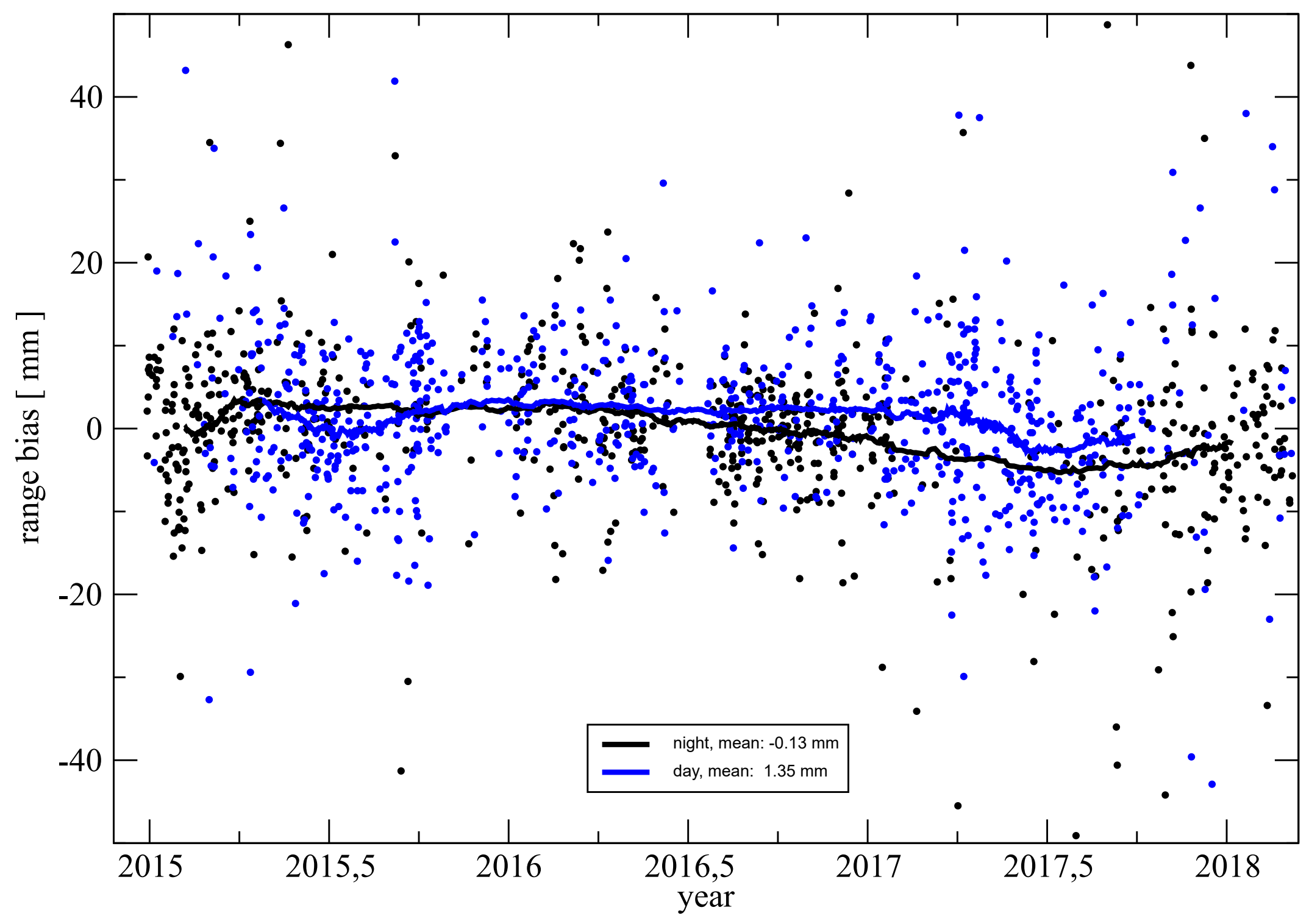


Fig. 8
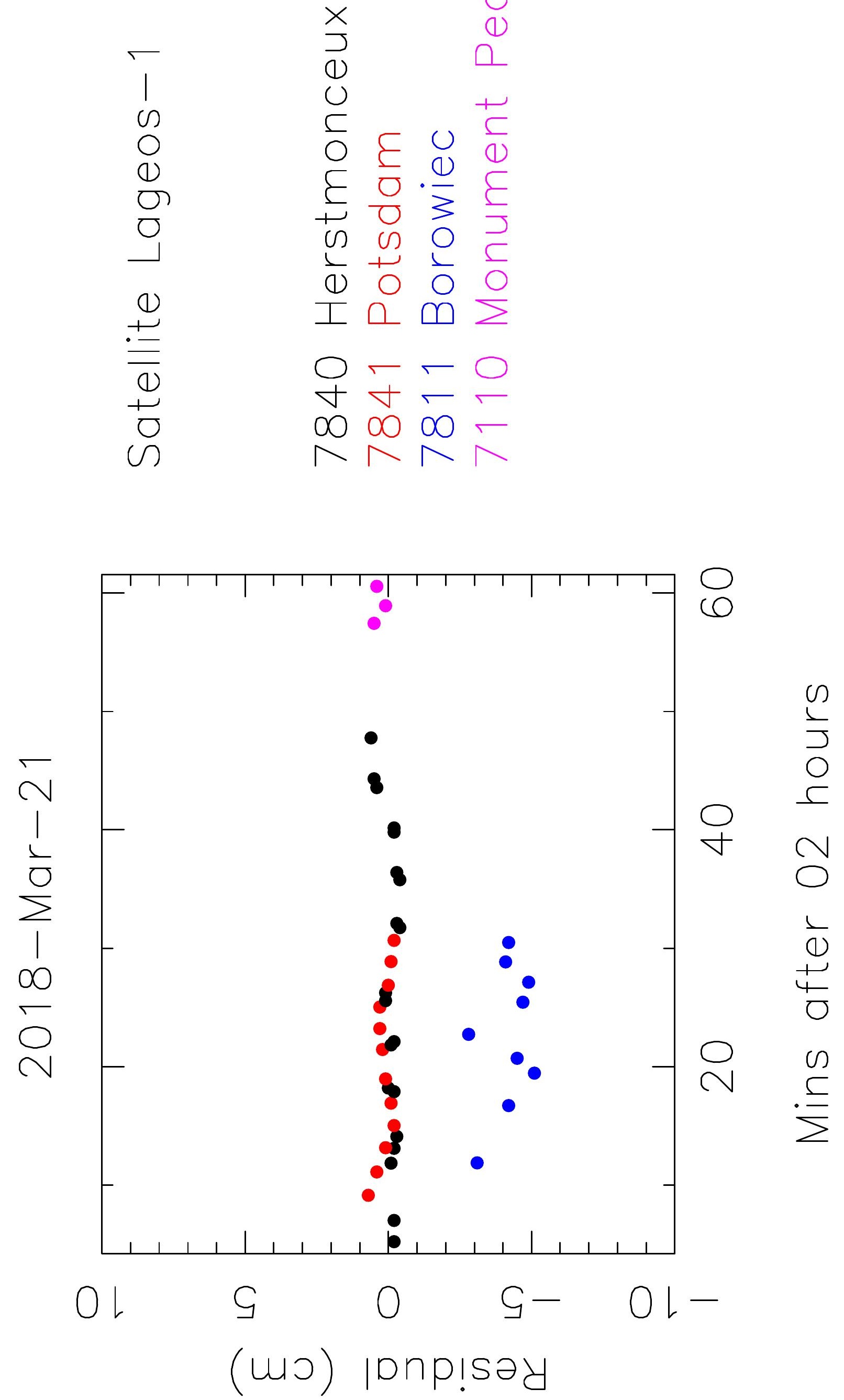


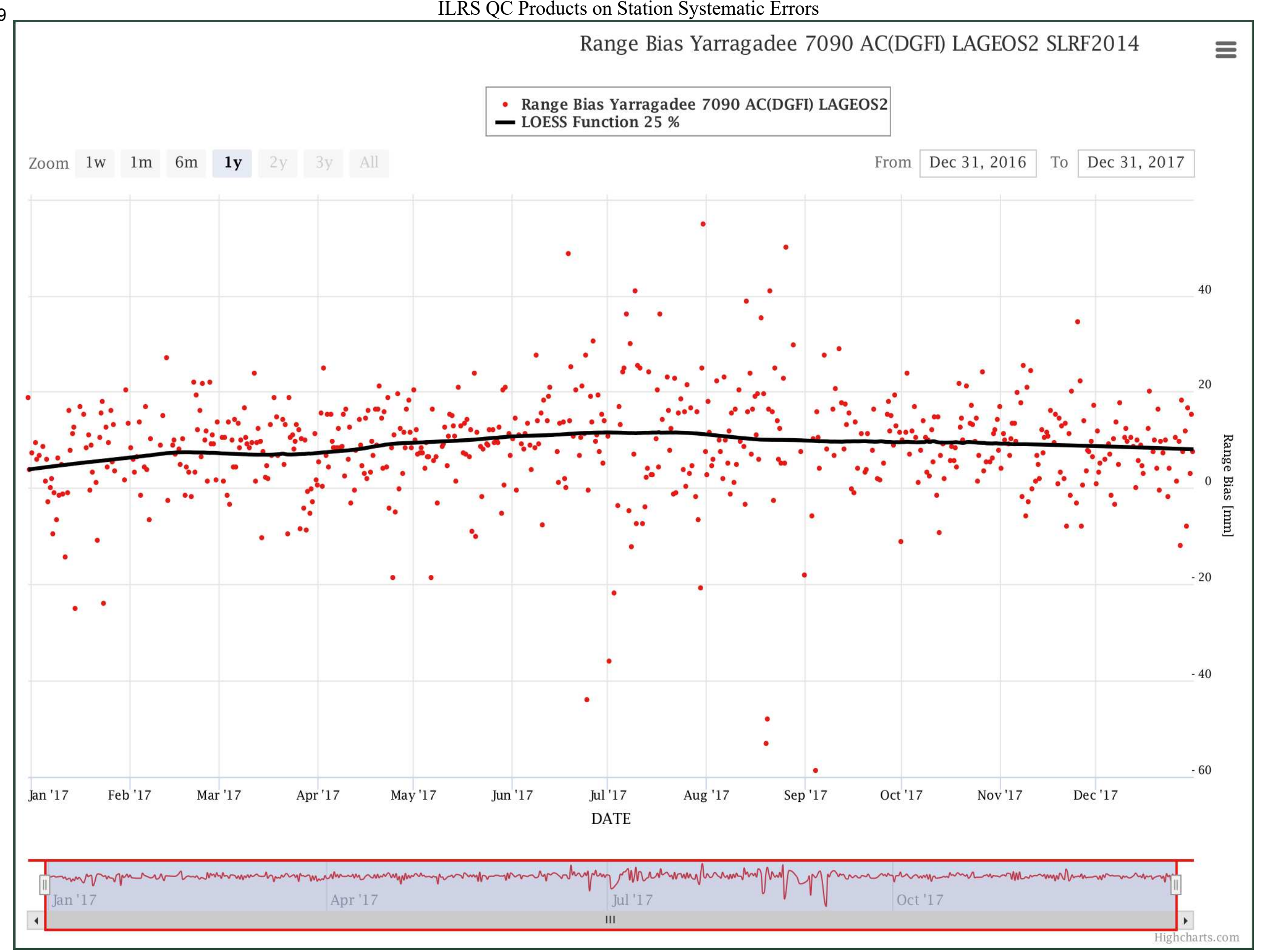

7090 Range Bias Yarragadee 7090 AC(DGFI) LAGEOS2

Mean/Std. Dev.: $9.01 \pm 16.11$ Count: 2,023

䙹




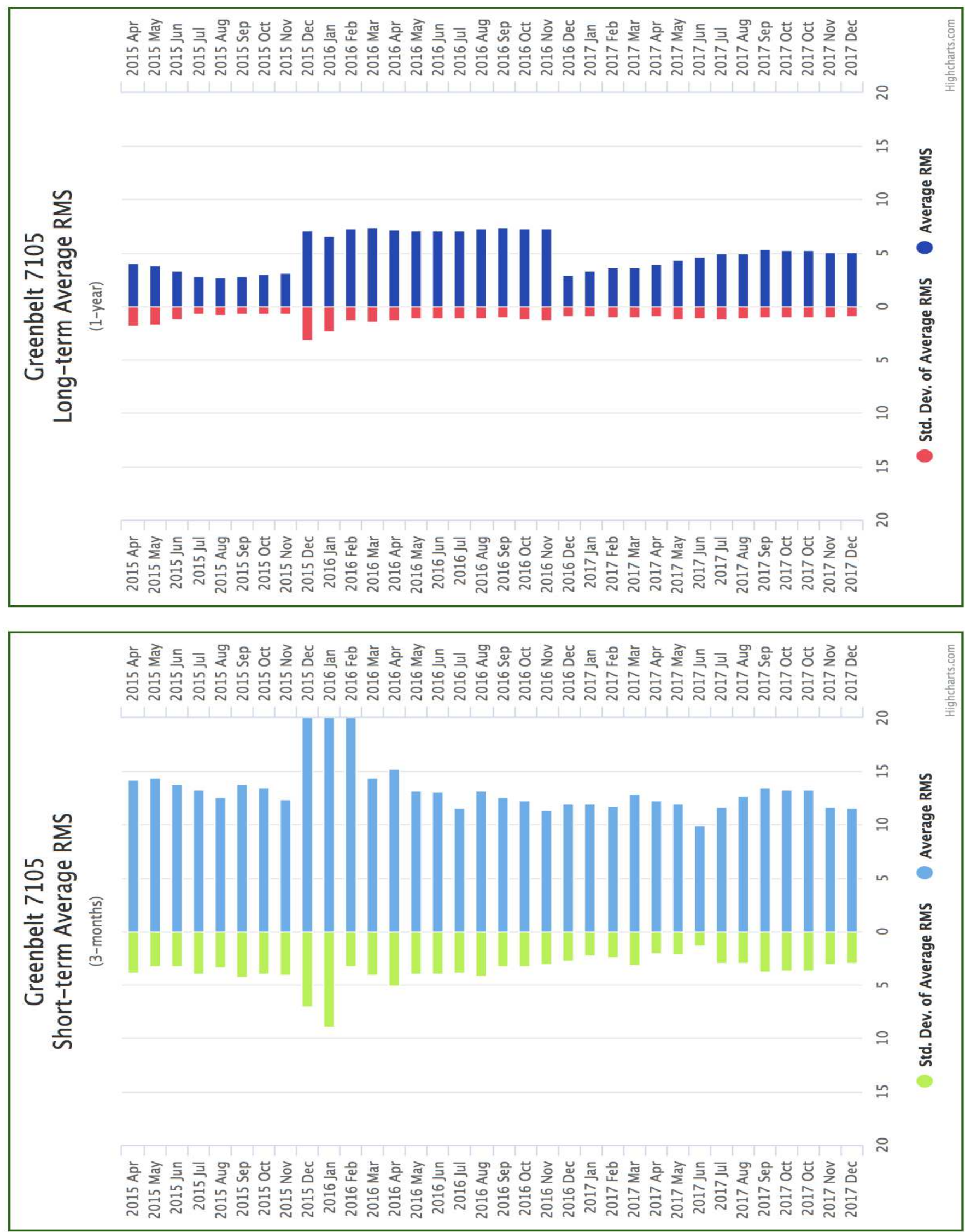

